\title{
$\mathrm{SRPH}$
}

Original Article

\section{System Level Simulation of Energy-Detection Based UWB Receivers}

\author{
Mostafa Yargholi* \\ Department of Computer and \\ Electrical Engineering, University of \\ Zanjan, Zanjan, Iran.

\section{*Corresponding Author: \\ \yargholi@znu.ac.ir}

Received: 10 September, 2020

Accepted: 30 October, 2020

Published: 30 November, 2020

\begin{abstract}
The performance of a non-coherent UWB receiver with Binary pulse position modulation is simulated with MATLAB; taking into account the effect of nonlinearity, noise, pulse shape and channel effects. This simulation examines the minimum requirements for LNA, AGC, squarer, and operational transconductance amplifier in analog front-end for sensor network application with $100 \mathrm{~Kb} / \mathrm{s}$ data rate and $10^{-3} \mathrm{BER}$. The linearity requirement in OTA is achieved using Gilbert cell OTA with the technique of multiple gated transistors. For sensor network applications, analog front-end modules must have $4 \mathrm{~dB} \mathrm{NF}$ (Noise figure), $-12 \mathrm{dBm} \mathrm{IIP} 3,50 \mathrm{~dB}$ gain and $-75 \mathrm{dBm}$ sensitivity for $100 \mathrm{~Kb} / \mathrm{s}$ data rates. The transceiver power consumption is assumed to be below $50 \mathrm{~mW}$. The performance of energy detection non-coherent receiver is simulated in Simulink of MATLAB, it shows that BER of Gaussian pulse is lower than doublet and $4^{\text {th }}$ Gaussian pulse. By increasing the number of transmitted pulse per bit and IIP3, the performance of receiver is improved.
\end{abstract}

Keywords: Non-coherent receiver, BER, OTA, Squarer, CM

\section{Introduction}

Energy detection Non-coherent UWB receivers [1, 2] are used in wireless sensor networks, which require devices with low complexity, low cost, and low power consumption. Sensor network receivers $[3,4]$ are used for location and ranging in indoor environments.

In $[5,6]$ system level of energy detection non-coherent receiver with VHDL-AMS hardware description language was presented. In [7] the implementation of low complexity detection, synchronization, and decoding algorithms for a non-coherent UWB receiver was explained. In $[8,9]$ circuit design of a Gaussian monocycle pulse generator and an UWB-IR noncoherent energy detection receiver consisting of LNA and digital baseband was presented.

A system-level simulation for energy detection noncoherent receiver was performed; taking into account the effect of $3^{\text {rd }}$ order nonlinearty, slew rate, and noise. Simulations are performed to analyze the receiver performance versus non-ideal parameters, SNR of channel and data rate. The minimum requirements for analog frond-end blocks are examined and the circuit structure and the techniques for linearity improvement are discussed. Linearity improvement in OTA with second order transconductance derivative (gm") cancellation with multiple gated transistors [10] is presented. Linearity improvement in Gilbert cell Squarer is investigated by utilizing modified superposition scheme [11] to cancel out both the second and third-order nonlinear currents.

\section{Energy detection based transceiver}

The architecture of energy detection based UWB transceiver illustrates in Figure 1. Assume binary pulse position modulation (BPPM) with rate $1 / \mathrm{T}$. Symbol "zero" corresponds to transmitting a pulse in the first half of the interval $(0, T)$, while symbol "one" corresponds to transmitting a pulse in the second half. Received signal is given by $[6,12]$ :

$$
r(t)=\sum_{k=-\infty}^{\infty} s\left(t-j T_{s}-a_{k} \frac{T_{s}}{2}-\tau\right)+n(t)
$$

$\mathrm{s}(\mathrm{t})$ is channel response to transmitted pulse; $\mathrm{a}_{\mathrm{k}}$ is Binary data, $T_{s}$ is symbol period, and $n(t)$ represents 
white Gaussian noise (AWGN) [12]. If $a_{k}=0$, the pulse is in the first half; if $a_{k}=1$, the pulse is in the second half of interval. The output data $\left(\mathrm{Z}_{\mathrm{k}}{ }^{(\mathrm{i})}\right)$ is given by:

$$
Z_{k}^{(0)}=\int_{k T_{s}+\tau}^{k T_{s}+\tau+\Delta} r^{2}(t) d t
$$

$$
Z_{k}^{(1)}=\int_{\left(k+\frac{1}{2}\right) T_{s}+\tau}^{\left(k+\frac{1}{2}\right) T_{s}+\tau+\Delta} r^{2}(t) d t
$$

It means that, the incoming waveform is first squared and then fed to an integrator-and-dump (I\&D) circuit; $\Delta$ represents channel spread [13]. If $Z_{k}^{(0)}>Z_{k}^{(1)}$ output binary sets to " 0 " ( $\hat{a}=0)$, otherwise $\hat{a}=1$.

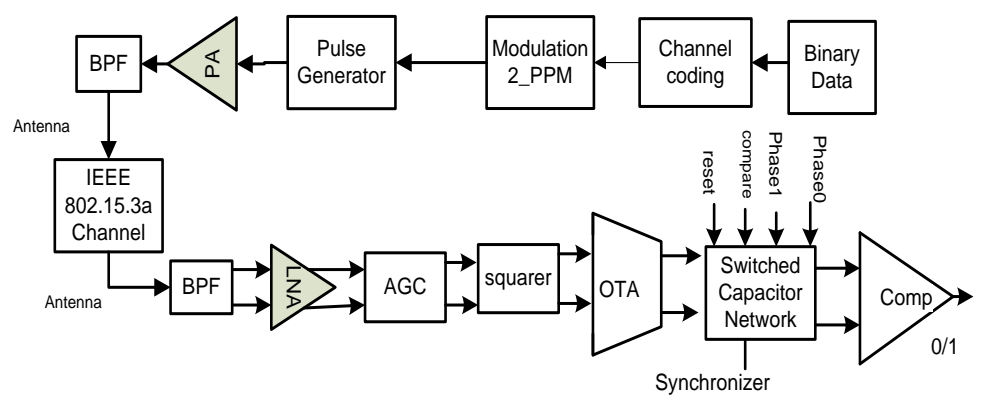

Figure 1. Transceiver architecture based on energy detection.

IEEE802.15.3a standard [14] as a typical multipath channel model for UWB systems is given by [15]:

$$
h(t)=\sum_{m} \sum_{l} a_{m l} \delta\left(t-\tau_{m}-\tau_{m l}\right)+n(t)
$$

where $a_{\mathrm{ml}}$ is amplitude of reflected signals, $\tau$ is path delay, and $\mathrm{n}(\mathrm{t})$ represents AWGN. The channel models $[16,17]$ are categorized as CM1-CM4, depending on the distance and line of sight condition.

\section{Stem level simulation}

An energy detection (ED) non-coherent UWB receiver is simulated with BPPM modulation for CM1-CM4 channel models. Transmitter is composed of channel coding, modulation block, UWB pulse generator and power amplifier. Receiver consists of LNA, AGC, squarer module, integrator (I\&D) and synchronizer [12].

System level simulation of transceiver is performed in the Simulink/Matlab. Requirements of analog front- end modules are achieved for $10^{-3}$ BER. For sensor network application, analog front-end modules must have $4 \mathrm{~dB} \mathrm{NF}$ (Noise figure), $-12 \mathrm{dBm}$ IIP3, $50 \mathrm{~dB}$ gain and $-75 \mathrm{dBm}$ sensitivity for $100 \mathrm{~Kb} / \mathrm{s}$ data rate. The transceiver power consumption is assumed to be below $50 \mathrm{~mW}$. The required performance of analog front-end modules are listed in Table1.

BER versus SNR for Gaussian pulse shape and its derivatives is shown in Figure 2. Gaussian pulse has more energy than its derivatives, and hence the BER of Gaussian pulse is lower than doublet and the $4^{\text {th }}$ derivatives of Gaussian pulse.

Figure 3 illustrates the effect of the number of pulse per bit (pulse/bit) on receiver performance with Gaussian pulse. The above figures show that by increasing the number of pulse per bit, the system performance is improved at the cost of decreasing transmission capacity. Figure 4 shows the effect of channel (CM1, CM2, CM3 and CM4) on BER of ED receiver.

Table 1

Required performance of analog front-end modules.

\begin{tabular}{lccccc}
\hline Module & $\begin{array}{c}\text { Gain } \\
(\mathbf{d B})\end{array}$ & $\begin{array}{c}\text { NF } \\
(\mathbf{d B})\end{array}$ & $\begin{array}{c}\text { B.W } \\
\mathbf{G H z}\end{array}$ & $\begin{array}{c}\mathbf{I I P 3} \\
\mathbf{( d B m})\end{array}$ & $\begin{array}{c}\mathbf{P}_{\text {diss }} \\
(\mathbf{m W})\end{array}$ \\
\hline LNA & $15 \sim 20$ & 2.3 & $3 \sim 5$ & -10 & 5 \\
AGC & DR=50 & 5 & $3 \sim 5$ & 8 & 8 \\
Squarer & 10 & --- & $3 \sim 5$ & 15 & 3 \\
OTA & 0 & ---- & $10 \mathrm{MHz}$ & 15 & 2 \\
\hline
\end{tabular}




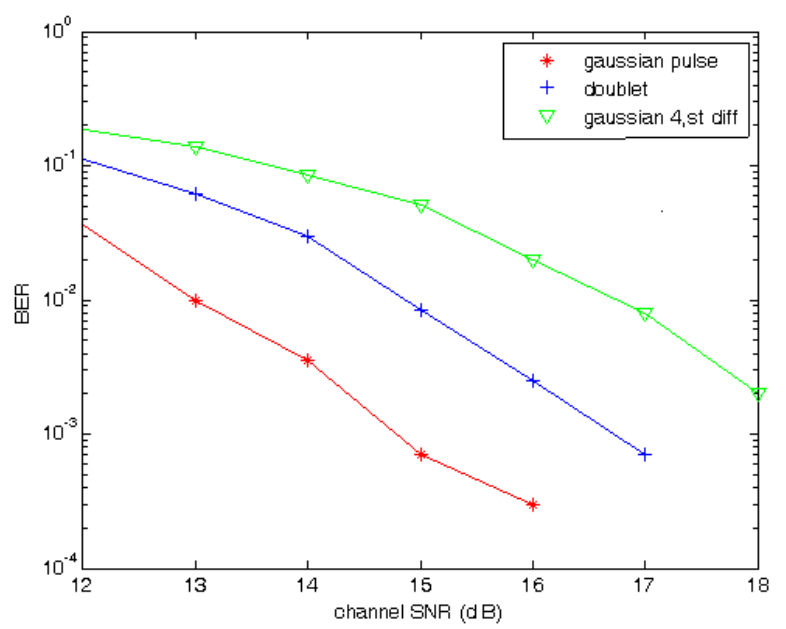

Figure 2. Transmitted pulse shape effect on receiver performance with 5 pulse/Bit.

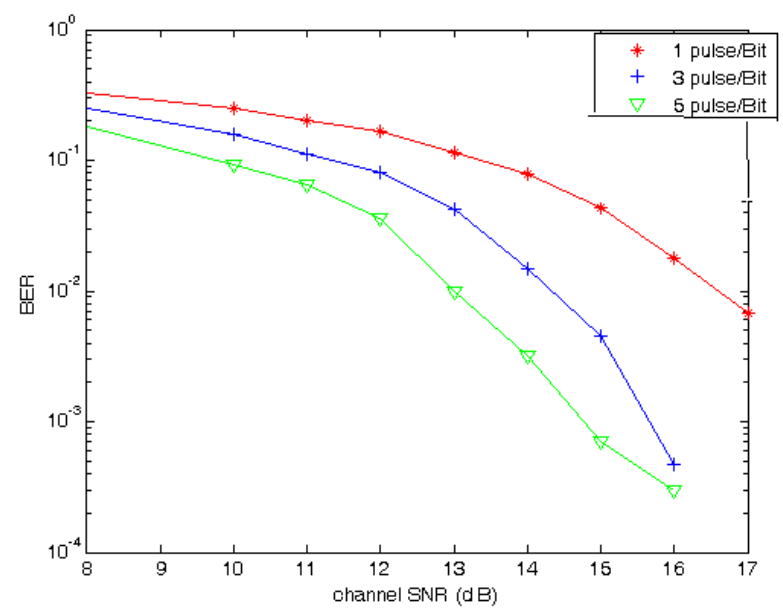

Figure 3. The effect of the number of pulse per bit on receiver performance for CM1 channel model.
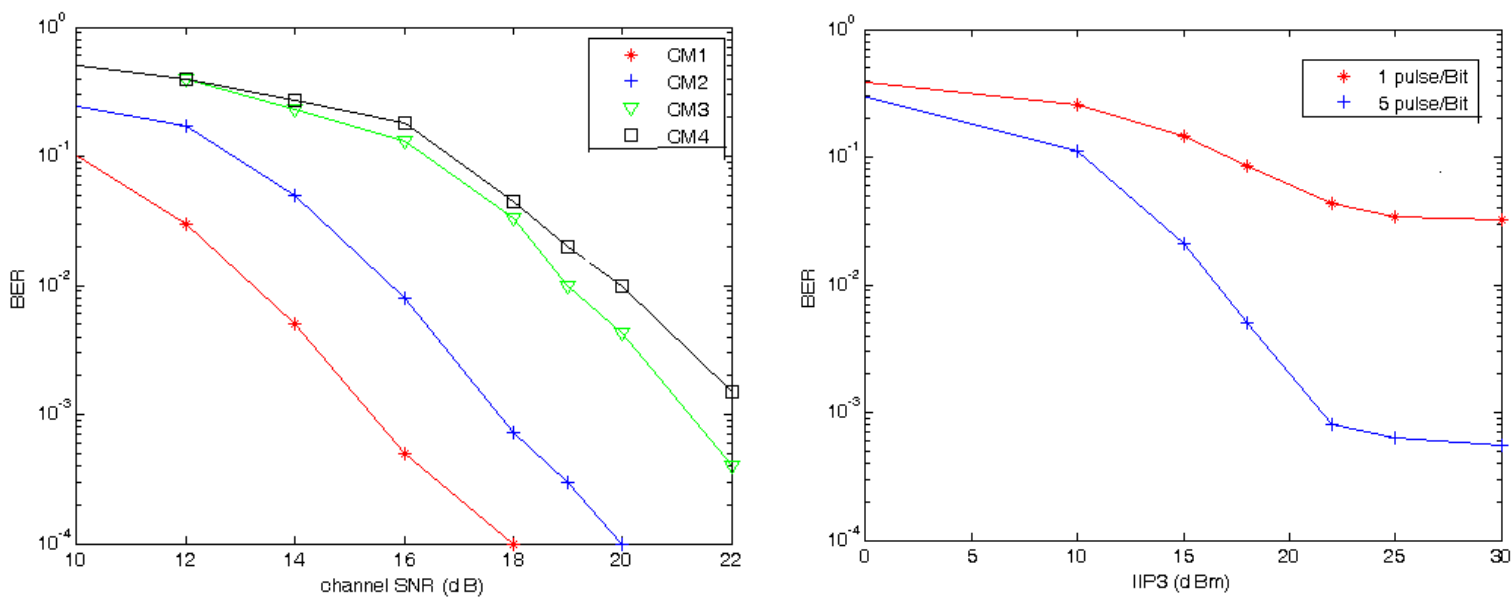

Figure 4. Channel effect on BER of ED receiver for 5pulse/Bit. Figure 5.The effect of Non-linear coefficient on BER.

The effect of $3^{\text {rd }}$ order non-linear parameters on receiver performance is shown in Figure 5. This figure shows BER versus IIP3. By increasing IIP3, receiver performance will improve. Due to BER sensitivity to non-linear effects, linearization techniques at circuit level are critical in receiver design. In the next section we describe the design of a highly linear OTA and squarer block. 


\section{Integrator for energy detection}

In order to design the integrator block, an OTA (operational transconductance amplifier) is required with $20 \mathrm{dBm}$ of linearity and power consumption around $1 \mathrm{~mW}$ [1].

To achieve the required performance, LNA needs a gain of $19 \mathrm{~dB}$, and power consumption below $5 \mathrm{~mW}$. s11 and s22 should be below -10dB. In addition, a squarer module is required with $10 \mathrm{~dB}$ gain, $20 \mathrm{dBm}$ IIP3, 95dBm IIP2 and power consumption around $1 \mathrm{~mW}$. Squarer based on Gilbert cell with intrinsic (second and third) distortion cancellation with PMOS auxiliary transistor is the best choice for this receiver. By using common gate structure in lower tree of Gilbert cell with flipped voltage followers [18], wide operation bandwidth is achieved.

Three common architectures of differential OTAs are: fully differential (FD), pseudo-differential (PD) and Gilbert cell. FD is typically based on a differential pair with a tail current source [19]. Disadvantage of a PD circuit is that, the common-mode and differential gain
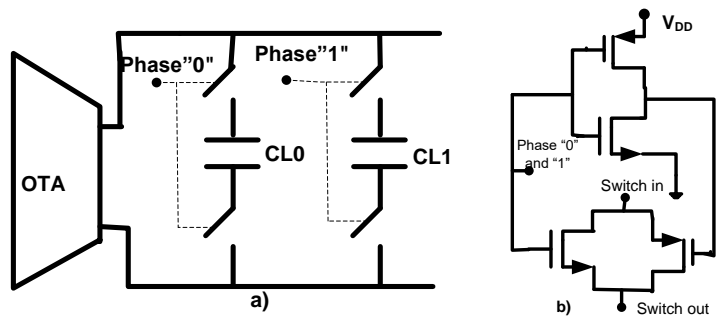

Figure 6. a) Gm-C integrator b) MOS switch scheme

\section{Conclusion}

The performance of energy detection non-coherent receiver is simulated in MATLAB. Simulation results show that Gaussian pulse BER is lower than doublet and $4^{\text {th }}$ Gaussian pulse. By increasing the number of transmitted pulse per bit and IIP3, system performance is improved. System level simulation of a non-coherent receiver with desired squarer and OTA, illustrates improvement of BER from $3 \mathrm{e}^{-2}$ to $6 \mathrm{e}^{-4}$.

\section{References}

1.Yargholi M, Nabavi A. Analog front end modules design in non-coherent UWB receivers for sensor networks. IEEE Conference on Semiconductor Electronic (ICSE2008), 2008; 22-23.

2. Donlan BM. Ultra-wideband narrowband interference cancellation and channel modeling for communications. M.Sc Thesis, Virginia State University, Jan. 2005.

3. Foerster JR. The performance of a direct-sequence spread spectrum ultra-wideband system in the presence is the same. Differences between the two signal paths, arising from device mismatch, will propagate to output. OTA based on Gilbert cell with third order distortion cancellation is a proper choice for energy detection receivers because of its linearity [20, 21]. In this paper, we examine the second order transconductance derivative (gm") cancellation with multiple gated transistors for linearity improvement in OTA.

$\mathrm{Gm}-\mathrm{C}$ architecture has high frequency (Parasitic can be absorbed to Capacitors), high tunability, high linearity and low power dissipation. Thus, Gm-C integrator is suitable for energy detection receivers because of linearity and high frequency response [19]. Switched capacitor as shown in Fig.6a is used to integrator design. A model of MOSFET switch is demonstrated in Fig.6b. Output voltage of capacitors CL0 and CL1 feed to a comparator to decide on output bits. Fig.6c shows Phase " 0 " control signal of switched capacitor. Phase " 1 " is in 40 ns to 80 ns and comparator control signals are in $85-100 \mathrm{~ns}$ for 10Mpulse/bit (maximum rate for non-coherent UWB receivers).

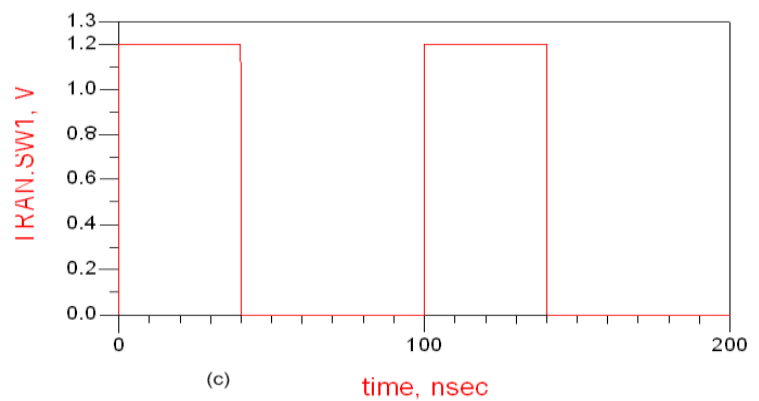

of multipath, narrowband, interference, and multiuser interference. IEEE Conference on Ultra Wideband Systems and Technologies, May 2002.

4. Nekoogar M. Introduction to ultra-wideband communications. Ultra-Wideband Communications, Fundamentals and Applications, Nekoogar. Book, Chapter 1, Aug, 2005.

5. Casu MR, Crepaldi M, Graziano M. A VHDL-AMS simulation environment for an UWB impulse radio transceiver. IEEE Transactions on Circuits and Systems, 2008; 55: 1368-1381.

6. Crepaldi M. Analysis, design and simulation of an UWB receiver for indoor localization. P.h.D Thesis, Politecnico di Torino, Italy, Nov 2005.

7 Vitavasiri S. A non-coherent ultra_wideband receiver: Algorithms and digital implementation. M.Sc Thesis, Massachusetts Institute of Technology, U.S.A, May 2007.

8. Stoica L. Non-coherent energy detection transceivers for ultra wideband impulse radio systems. P.h.D Thesis, University of Oulu, Finland, Feb 2008. 
9. Bo-Ting Wang S. Design of ultra-wideband RF front-end. P.h.D Thesis, California, Berkeley, USA, Fall 2005.

10. Yargholi M, Nabavi A. A highly linear Gilbert cell OTA with multiple gated transistors for non-coherent UWB receivers. IEICE Electronic Express, Integrated Circuit, 2009; 11(6): 756-762.

11. Parvizi M, Nabavi A. Highly linear common-gate mixer employing intrinsic second and third order distortion cancellation. IEICE Electronic Express, Integrated Circuit, 2009; 6(6): 310-316.

12. Crepaldi M, Casu MR, Graziano M. Energy detection UWB receiver design using a multi-resolution VHDL-AMS description. IEEE, Signal Processing Systems Design and Implementation, 2005; 13-18.

13. Stoica L, Tiuraniemi S, Oppermann I, Repo H. An ultra wideband low complexity circuit transceiver architecture for sensor networks. IEEE, Confrence, 2005; 364-367.

14. Revision of part 15 of the commission's rules regarding ultra-wideband transmission systems. First note and Order, Federal Communications Commission, ET-Docket 98-153, Adopted February 14, 2002, released April 22, 2002. Available: http://www.fcc.gov/Bureaus/Engineering

Technology/Orders/2002/fcc02048.pdf

15. Sheng $H$. Transceiver design and system optimization for ultra-wideband communication. ph.d
Thesis, Department of Electrical and Computer Engineering, NJIT, May 2005.

16. Choi JD, Stark WE. Performance of ultrawideband communications with suboptimal receivers in multipath channels. IEEE J Select Area Comm. 2002; 20: 17541766.

17. Idriss A, Schmidt A, Zeisberg S, Finger A. Performance of a non-coherent receiver for UWB communication and ranging applications. IEEE Info Comm Technol. 2006; 2314-2319.

18 Carvajal RG, Ramirez-Angulo J, Lopez-Martin AJ, Torralba A, Galan JAG, Carlosena A, Chavero FM. The flipped voltage follower: A useful cell for lowvoltage low-power circuit design. Circuits and Systems I: Regular Papers, IEEE Transact. 2005; 7(52): 12761291.

19. Ghabrial W. Transconductance-C filter design for wireless telecommunications receivers. P.h.D Thesis, Electrical and Computer Engineering, Carnegie Mellon University, 2003.

20. Yargholi M. A highly linear squarer design for energy-detection RF receivers. Microelect J. 2013; 44: 658-662.

21. Mohieldin AN, Sánchez-Sinencio E, Silva-Martínez J. A low-voltage fully balanced OTA with common mode feedforward and inherent common mode feedback detector. ESSCIRC, Texas A\&M University, USA, 2002; 191-194.

\section{SJIS}

Copyright: (C) 2020 The Author(s); This is an open-access article distributed under the terms of the Creative Commons Attribution License (http://creativecommons.org/licenses/by/4.0), which permits unrestricted use, distribution, and reproduction in any medium, provided the original work is properly cited.

Citation: Yargholi M. System Level Simulation of Energy-Detection Based UWB Receivers. SJIS, 2020; 2(4): 10-14.

https://doi.org/10.47176/sjis.2.4.10 